\title{
KN03 | SOlar System SeCRETS Hidden In QuAsicRYSTAlS
}

Bindi, Luca (University of Florence, Firenze, ITA)

Quasicrystals are solids whose diffraction patterns are composed of diffraction peaks, like periodic crystals, but with symmetries forbidden to crystals. Natural quasicrystals (icosahedral and decagonal) were discovered a decade ago. The search took more than a dozen years and has opened a new frontier in mineralogy that could lead to new discoveries in geoscience, astronomy, condensed matter physics, and materials engineering. For the first time, minerals have been discovered that violate the symmetry restrictions of conventional crystallography. That nature could accomplish this without human intervention was unexpected, requiring the existence of petrological processes never considered previously. The fact that the quasicrystals were found in a meteorite formed in the earliest moments of the solar system means these processes have been active for over 4.5 billion years and influenced the mineral composition of the first objects to condense around the Sun. Finding quasicrystals formed in these extreme environments informs the longstanding debate about the stability and robustness of quasicrystals among condensed matter physicists. Recent shock experiments, with starting materials similar to the intermetallic alloys in the meteorite hosting the quasicrystals, lend support to the hypothesis that the extra-terrestrial quasicrystals formed as a result of hypervelocity impacts among objects in the early Solar system. Finally, the discovery inspired further searches for quasicrystals and other new forms of matter not seen in the laboratory previously which may provide valuable new materials for physics and engineering. This culminated in the discovery of an icosahedral phase with a composition not predicted before in laboratory experiments. 